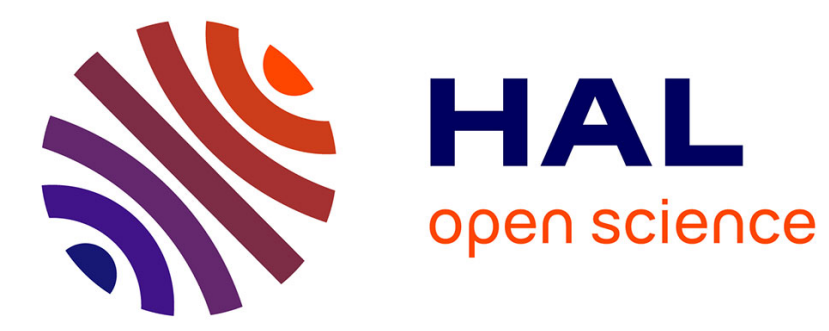

\title{
Practical Performance Comparison of 1-D and 2-D Decoding Methods for a Chipless RFID System in a Real Environment
}

Raphael Tavares de Alencar, Zeshan Ali, Nicolas Barbot, Marco Garbati, Etienne Perret

\section{To cite this version:}

Raphael Tavares de Alencar, Zeshan Ali, Nicolas Barbot, Marco Garbati, Etienne Perret. Practical Performance Comparison of 1-D and 2-D Decoding Methods for a Chipless RFID System in a Real Environment. IEEE Journal of Radio Frequency Identification, 2020, 4 (4), pp.532-544. 10.1109/JRFID.2020.2997988 . hal-03450581

\section{HAL Id: hal-03450581 https://hal.science/hal-03450581}

Submitted on 26 Nov 2021

HAL is a multi-disciplinary open access archive for the deposit and dissemination of scientific research documents, whether they are published or not. The documents may come from teaching and research institutions in France or abroad, or from public or private research centers.
L'archive ouverte pluridisciplinaire HAL, est destinée au dépôt et à la diffusion de documents scientifiques de niveau recherche, publiés ou non, émanant des établissements d'enseignement et de recherche français ou étrangers, des laboratoires publics ou privés. 


\title{
Practical Performance Comparison of 1-D and 2-D Decoding Methods for a Chipless RFID System in a Real Environment
}

\author{
Raphael T. Alencar, Student Member, IEEE, Zeshan Ali, Nicolas Barbot, Marco Garbati \\ and Etienne Perret, Senior Member, IEEE
}

\begin{abstract}
A comparison of four decoding methods for a Chipless RFID system was executed by analyzing the read success rate of a frequency-position coding tag with 12-bit of capacity. Scattering parameter measurements have been performed in a real environment, using a fixed antenna, with the tag moving within a $30 \times 30 \times 30 \mathrm{~cm}^{3}$ volume. Optimal parameters have been obtained based on the decoding success rates of the practical measurements, for background calibration, time-gating, Short Time Fourier Transform (STFT) and Short Time Prony Analysis (STPA) methods. STFT and time-gating showed the best volume reading performance. Time-gating has been found to be the best decoding method for the chipless RFID system employed, as it presented the best performance amongst the other methods while being a less complex, 1-D, approach.
\end{abstract}

Index Terms - Chipless RFID, Measurement, Prony, RCS, Reading Volume, Spectrogram, STFT, Tag Characterization, Time-Gating, UWB.

\section{INTRODUCTION}

$\mathrm{R}_{\mathrm{i}}$ FID technology is an ever-advancing subject, with an increasing range of applications, and its use is interesting for automatic identification and tracking systems, which can be seen in a variety of domains, such as warehousing, packaging [1] wholesale, retail, security, military, post offices, toll collection, ticketing, smart cards, smart documents, access control, health care, pharmaceutical [2], [3], library management, among others [4]-[9]. However the majority of these applications are mostly implemented using barcodes, QR codes and magnetic cards [10].

Besides automatic identification and tracking, RFID is consolidating itself in the sensor domain, as RFID tags allow for measuring several different physical parameters, and detecting variations in the environment [11], their use is of interest for various domains which profit from wireless sensors, such as temperature, humidity and gas sensors,

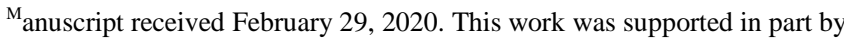
the European Research Council (ERC) under the European Union's Horizon 2020 Research and Innovation Program (grant agreement No 772539).

R. T. Alencar, Z. Ali, N. Barbot and M. Garbati are with the Univ. Grenoble Alpes, Grenoble INP, LCIS, 26000 Valence, France (e-mail: raphael.tavares-de-alencar@lcis.grenoble-inp.fr).

E. Perret is with the Univ. Grenoble Alpes, Grenoble INP, LCIS, 26000 Valence, France, and also with the Institut Universitaire de France, 75005 Paris, France.
}

industrial robotics [6], videogames [12], precision medicine [13], precision agriculture [14] and more. The domain of Internet of Things (IoT) is one in which the sensor and identification characteristics of RFID become very interesting, and it has generated a great amount of attention towards the technology thanks to the advantages it presents in comparison with others [6]. Nonetheless, a limiting factor to the dominance of RFID is the cost of the tag, primarily linked to the cost of the chip considering the fact that a traditional passive RFID tag costs around US\$ $0.15-0.5$ to be produced [15]-[17].

In the context of different classes of tags being needed for different applications, in regard to their particular specifications (capacity, size, cost, reliability, security...), Chipless RFID technology presents characteristics that make it an attractive solution for several automatic detection, identification and sensing purposes. It allows non-line-of-sight reading and sensing capability, it can have read ranges of up to $1 \mathrm{~m}$. The fabrication of Chipless tags presents a competitive cost - estimated to be US\$ 0.005 for large scale production since it does not have a silicon microchip embedded into it [16], [17]. Finally, it provides a relatively high robustness in harsh environments when compared to traditional RFID [9], [18].

To implement a Chipless RFID system in an industrial environment, a complete system is needed, with robust decoding capacity, while taking international regulations into account [18].

In the literature, most of the attention is turned onto the tag design and characteristics, for example: tags with depolarizing characteristics, aiming to reduce the effect of environment reflections [19]-[21]; compact tag designs for small surfaces [22]-[25]; tags that are rotation-independent and can be read at any orientation with respect to the reader [21], [26], [27]; tags with high coding capacity [16], [22], [23]; printable tags, which have potential to be very inexpensive and marketable [6], [28]-[31]; tags with sensor capabilities such as rotation sensing, structural health monitoring for buildings and sensing of multiple environmental and material parameters [20], [32][36]; alongside different tag designs with specific intents such as to mimic QR codes or alphabetic letters [31], [37].

However, the performance of a chipless system still depends on the decoding algorithm used by the reader to 
decode the ID of a tag. This paper evaluates the performance of the classical post-processing algorithms available in the literature to decode a Chipless RFID tag from the same dataset. Our approach has, therefore, been a practical one, in which we compare the different decoding techniques in terms of overall reading success rate for a tag displaced inside a given volume.

This paper is an extension of [38], in which two decoding methods for cross-polarization Chipless RFID tags were compared, based on the tag's overall detectability and correct decoding as it was placed and measured in 29,791 different positions within a $30 \times 30 \times 30 \mathrm{~cm}^{3}$ volume, with a monostatic cross-polarization setup. Here the work is extended by applying four different types of decoding methods to the extensive set of measurements. Each method can be categorized as a 1-D or 2-D approach, in terms of how the information is decoded.

The methods vary in complexity, the 1-D methods being less complex than the 2-D methods. Decoding with 1-D methods analyzes the frequency-domain (FD) values of the $S_{21}$ parameter measured, and for these, the amplitude FD representations show the peaks corresponding to the resonances of the tag. In the 2-D methods, the measurement is represented in the form of spectrograms or histograms.

The decoding methods analyzed are:

1. Background Calibration - subtraction of an empty reference measurement (i.e., measured in the absence of tag) and extraction of ID (identification) from the peak apexes of FD signal;

2. Time-Gating - in addition to the background calibration, use of a temporal window to discard the unwanted parts (i.e., reflection from tag's holder and surrounding objects) in TD signal and extraction of ID from the peak apexes of FD signal;

3. Short Time Fourier Transform (STFT) - use of STFT averaging on TD signal and extraction of ID from the peak apexes of averaged FD signal;

4. Short Time Prony Analysis (STPA) - use of STPA on TD signal and extraction of ID from the maximum probable bins of extracted poles' histogram.

We are able to determine the advantages and drawbacks in using each type of decoding, and we point out that the performance of STPA for chipless RFID tag detection has never been discussed before.

The paper is divided as follows: in Section II we describe the operation of a chipless RFID system, detailing how a chipless RFID tag can contain and transmit information, and the measurement bench set up for this work is also described. In Section III and IV, the different decoding approaches applied are detailed, dividing them into the two categories 1-D and 2-D; Section V is reserved for the results obtained and the analyses; and in Section VI, the conclusions about the study are drawn.

\section{ChiPLESS RFID AND MEASUREMENT SETUP}

\section{A. Chipless RFID}

A chipless RFID system works as follows: the reader, which operates following UWB regulations [18], generates and transmits a flat ultra-wideband (UWB) signal. Then, the signal reaches the tag and is backscattered carrying the tag's information. Finally, the reflected signal is received at the reader antenna and is decoded - the information corresponding to a unique identifier from a database. The tag in a chipless RFID system is fully passive, and it can be categorized according to the type of encoding to which it resorts: frequency-coding, time-coding, image-based or hybrid coding [21].

Frequency-coding (FC), also known as frequency- position coding, frequency-based and frequency-domain coding, corresponds to the way the information is embedded in the spectral signature of the tag. In this case, the tag produces either notches or peaks in the FD representation of the backscattered signal, depending on the tag geometry. These types of tags convey information by using the presence/absence of the resonances in determined positions as, for example, ' 1 ' $/{ }^{\circ} 0$ ' in a binary code [19]-[40]. This is the type of tag used in this work.

Time-coded (TC), time-based, or time-domain reflectometry (TDR) tags, in the other hand, code information through the use of the multiple time-delayed reflections produced in the backscattered signal in the time-domain (TD) [41], [42].

In Image-based and spatial-based chipless RFID, the information is coded with use of the relative position between the resonators, in order to identify the tag through its form, rather then its resonant frequency. For example, through the presence/absence of a resonator in a given position being read as ' 1 '/'0' -[43]-[48]; or by stacking different dielectrics, the EM-imaging of a multilayered dielectric structure can be used to identify an individual tag [49], [50].

Hybrid coding is achieved by combining two or more factors with the goal of increasing the information capacity of the tag [51]. The different parameters used for hybrid coding can be angle/polarization [25], [51], [52], phase [16], RCS magnitude [53], [54], attenuation level [55], and it can also be combined with a time-coding approach [56], [57]. The different types of hybrid coding can entail an increase of the complexity of the reader [46].

Since the chipless tag is a passive linear device with no RCS time modulation, when reading in a real environment, a number of difficulties arise:

1. The signal power backscattered from the tag is very low, and this is combined with the path loss which increases with the distance between the reader antenna and the tag;

2. Usualy, there is a strong coupling between the emitting and receiving signals at the reader front-end part;

3. The reflection from the tag contains not only the resonant signal conveying information, but also part of the interrogating signal is reflected back from the tag in a quasi-optical fashion, generating noise; 
4. The environment generates a lot of noise, such as EM interference and multipath fading, due to the objects and walls in the surroundings;

5. The interference due to the presence of wireless networks is also an issue inside an office environment.

To reduce the impact from the issues mentioned above, some techniques can be applied, most are done through signal post-processing.

Taking into account the two first issues, a depolarizing chipless RFID tag is used, which suppresses the deteriorating co-polarized interferences [19]. Since whenever a signal is transmitted in a given direction, it is not only reflected by the tag, but also gets reflected off the surroundings, resulting in a low signal-to-noise ratio (SNR). When performing a crosspolarization reading, the reader emits a signal with a given polarization (e.g. horizontal) and receives the signal in the orthogonal direction (in this case, vertical). Nonetheless, for the tag's spectral signature to be identified, the tag must be depolarizing, reveiving the horizontally-polarized signal and backscattering part of it in the vertical polarization.

To mitigate the coupling effects from the antenna, a calibration technique can be used. The calibration consists of performing an 'empty' measurement, without the presence of the tag, and subtracting it from the measurements with the tag [18], [19], as such

$$
S_{21}^{\text {backg.calib }}=S_{21}^{\text {tag }}-S_{21}^{\text {back }}
$$

in which $S_{21}^{\text {tag }}$ corresponds to the $S_{21}$ measured in presence of the tag, $S_{21}^{\text {back }}$ is the "empty" measurement, without the tag. This is known as background calibration, or environment subtraction.

As mentioned, part of the reader interrogating signal is specularly reflected back from the tag; this first scattering mechanism is called the tag's structure mode and depends only on the tag's geometry and materials. Another part of the energy is stored by the tag's structure and is backscattered following a process that extends in time and depends of the quality factor of the structure. This second phenomenon is the tag's antenna mode, and it is the mechanism that conveys information, through resonance [18], [19]. One way to counter this effect is through the use of time-gating (or timewindowing), where the TD signal backscattered from the tag is not completely used, but only a window of the complete TD signal is selected, which ideally should have a large portion of resonant content [58].

We point out that the frequency-encoded tag can be read with a reader operating in time or in frequency to get the same information [18]. In the case of a time-based reader, the reader receives the full TD signal which inherently contains the structure mode and the antenna mode, as well as additive background noise and noise due to multipath. The same is for a frequency-based reader, once applied the Inverse Fast Fourier Transform (IFFT) to the tag's response.

\section{B. Chipless RFID Tag}

The tag used for this work was the depolarizing tag introduced in [19], which can be seen in Fig. 1. It is based on RF encoding particles [40] and consists of eight independent resonators, where each resonator is composed of a set of five identical shorted dipoles, oriented at $45^{\circ}$. One resonance between $3 \mathrm{GHz}$ and $7 \mathrm{GHz}$ is associated to each resonator, and the code of the tag is determined by the allocation of all the resonances. The tag was realized with $35 \mu \mathrm{m}$-thick copper with a $0.8 \mathrm{~mm}$ RO4003 substrate $\left(\epsilon_{R}=3.55, \tan \delta=\right.$ $0.0025)$ and a copper ground plane. The total surface of the tag is $85 \mathrm{~mm} \times 53 \mathrm{~mm}$.

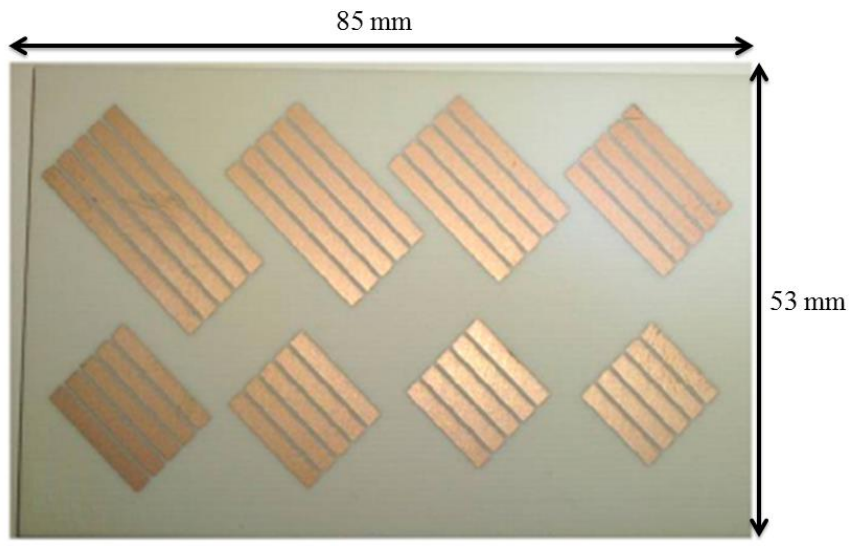

Fig. 1. Frequency-coded chipless RFID tag used [19].

An example of the FD signal backscattered from the tag can be seen in Fig. 2(b). The tag uses frequency-coding described as follows: the bandwidth from $3 \mathrm{GHz}$ and $6 \mathrm{GHz}$ is divided in 6 bands of $400 \mathrm{MHz}$ (vertical black lines in Fig. 2(b)), and each of these bands is divided in 4 sub-bands of $100 \mathrm{MHz}$ (dashed black lines). The decoding of the tag is done through the use of a script which, for every measurement performed, simply evaluates the position of the highest peak inside each band and, according to in which of the four sub-bands it is located, attributes a binary value for each resonance. Thus, any peaks besides the highest in each band are discarded and if a highest peak is seen in an 'incorrect' band, the code obtained will be erroneous.

We have considered only the six first resonators, due to the lower SNR observed for the higher frequencies for distances greater than $10 \mathrm{~cm}$.

The information capacity for an FC tag is given by the formula

$$
C=N \cdot \log _{2} F
$$

in which $F$ is the number of possible frequency slots per resonator, and $N$ corresponds to the number of resonators. Thus the coding capacity of the tag used in this study is

$$
6 \cdot \log _{2} 4=12 \text { bits. }
$$

As an example, the blue curve in Fig. 2(b) corresponds to the decoding with background calibration, whereas the red curve is the decoding without background calibration. The green rectangles indicate the 'correct' sub-bands for the used tag (which has ID $=00.00 .00 .00 .00 .00$ ). We can see that the signal with background calibration is correctly decoded at the given position, whereas the decoding without background calibration results in an error, with the $3^{\text {rd }}$ and $6^{\text {th }}$ resonant 
peaks being mistakenly detected in different sub-bands, indicated in red. The erroneous decoding yields ID = 00.00 .11 .00 .00 .10 .

\section{Measurement Bench}

The measurement setup is shown in Fig. 3. It was assembled in a real environment, surrounded by objects and walls in all directions, without use of any EM absorbent material.

The antenna used is a dual-polarization wideband openboundary quad-ridge horn (SATIMO QH2000). It operates in the $2-32 \mathrm{GHz}$ band and has gain varying from $6 \mathrm{dBi}$ to $11 \mathrm{dBi}$ inside our interest bandwidth, from 3 to $7 \mathrm{GHz}$. The antenna was positioned facing upwards on a fixed arm, in a monostatic configuration. The mechanical arm had a width of $5 \mathrm{~cm}$.

The frequency-based reader was emulated by using a vector network analyzer (VNA) FieldFox Handheld Microwave Analyzer (Keysight N9918A) with $0 \mathrm{dBm}$ output power. Port 1 of the reader was connected to the vertical polarization port of the antenna, and the port 2 was connected to the horizontal polarization port of the same antenna. So port 1 (vertical) operated in transmission while port 2 (horizontal) operated in reception, and the S21-parameter corresponds to a measurement in cross-polarization $(\mathrm{VH})$.

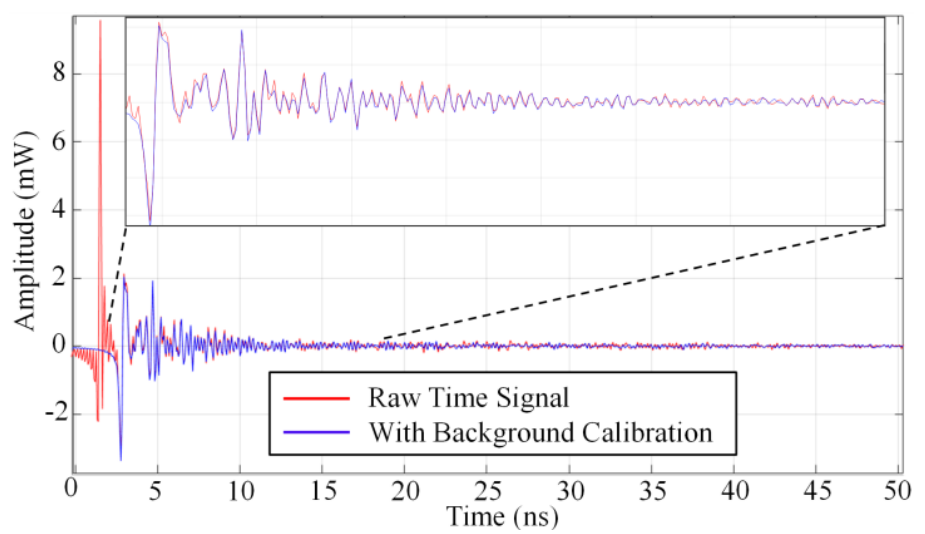

(a)

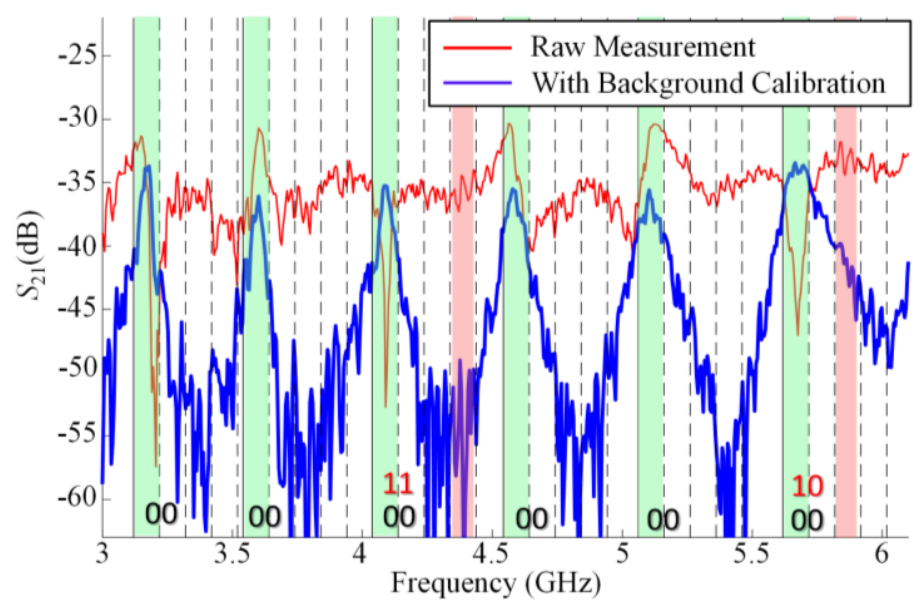

(b)

Fig. 2. (a) Difference between the raw time-domain signal (in red) and the background-calibrated signal (in blue) measured at position $(0,0,10) \mathrm{cm}$; (b) Frequency-domain representation, raw (in red) and background-calibrated (in blue);
The tag was placed facing downwards, on the end of a mechanical moving arm controlled by software (see Fig. 3). The tag's initial position was centered in front of the antenna, at a distance of $0.2 \mathrm{~cm}$. This is determined as the initial position of the tag, given by coordinates $\left(x_{i}, y_{i}, z_{i}\right)=(0,0,0)$.

The mechanical arm was displaced in steps of $1 \mathrm{~cm}$ in the $x-, y$ - and $z$-axes, once at a time, with one measurement being performed for each position inside the cube determined by $-15 \leq x \leq 15 ;-15 \leq y \leq 15 ; 0 \leq z \leq 30$ (in cm).

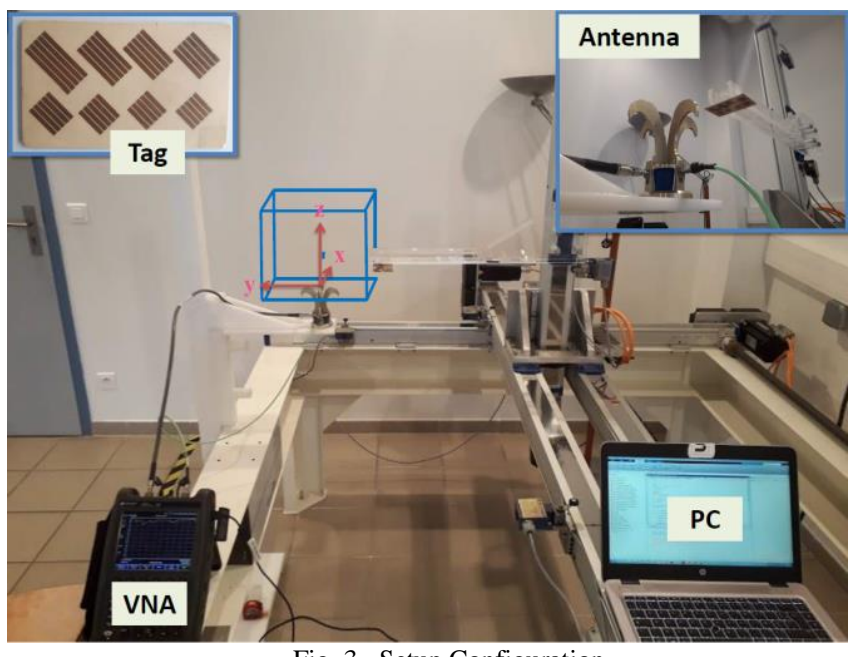

Fig. 3. Setup Configuration.

For each position, the response of the tag was measured and stored. A single "empty" measurement, without presence of the tag, was taken, to be used for the decoding techniques which perform background calibration. The campaign amounted to a total of 29,791 measurements.

The volume in which the tag was measured is represented by the blue box in Fig. 3, where a coordinate system (shown in red) is positioned at point $(0,0,0)$. A close-up view of the antenna and the tag attached to the mechanic arm is seen in the top-right corner. Both the VNA and the mechanical arm are controlled by a computer (PC).

All the measurements performed inside the described volume were decoded individually and automatically, following four methods, described in the following two sections. The same set of measurements was used, only the decoding process is changed.

\section{1-DIMENSIONAL DECODING TECHNIQUES}

In this Section, we present the implementation of two 1-D amplitude-based decoding approaches, i.e. background calibration and time-gating.

\section{A. Background Calibration}

As mentioned previously, background calibration can be used to reduce the coupling effects from the antenna. In the case presented, we have performed a single 'empty' $S_{21}$ measurement, without the tag, and with the arm's edge positioned in the center of the evaluated volume corresponding to position $\left(x_{e}, y_{e}, z_{e}\right)=(0,0,15)$ - and this same measurement is used for the background calibration of all the measurements with the tag in all the 29,791 positions. 
When applying more complex decoding techniques together onto a background-calibrated set of measurements (as presented in following subsections), the same single 'empty' measurement is used, as was presented here.

In Fig. 2(a) and 2(b) we can observe an example of a measurement performed at position $(0,0,10)$. In Fig. 2(a), we can see its TD representation, and that the background calibration eliminates the initial pulse present in the first $0.5 \mathrm{~ns}$ of the received signal, which is mostly due to the antenna coupling. In Fig. 2(b) we observe the FD representation, and it can be seen that, since the tag is centered in front of the antenna, the background calibration has a very positive impact on the detection of the tag's correct ID (correct frequency slots highlighted in green, as mentioned in Section II.B). We also observe that the average amplitude of the raw measurement is higher (about $+10 \mathrm{~dB}$ ), however, this is due to the antenna coupling, which increases the signal's power density but contains no information. This reduces the tag's detectability as it reduces the SNR and the frequency peaks from the raw measurement have a smaller dynamic range.

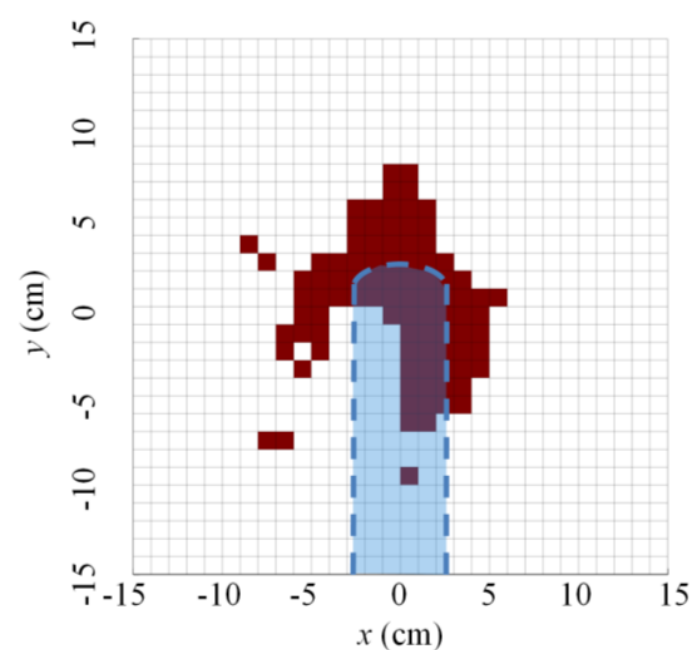

(a)

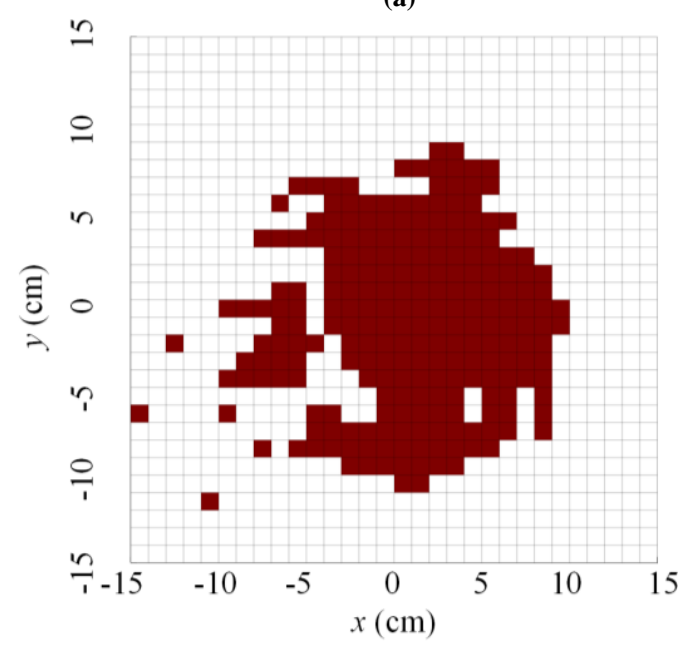

(b)

Fig. 4. Positions where the tag was successfully read (in red) for the same plane, at $z=10 \mathrm{~cm}$. (a) With background calibration; (b) with time-gating and background calibration.
Evidently, since the mechanic arm was displaced together with the tag, the background measurement did not take this into account and for this it adds error to the background calibration, especially for positions in which the arm is in front of the antenna.

In Fig. 4(a) we observe the $30 \times 30 \mathrm{~cm}^{2}$ plane (for distance $z=10 \mathrm{~cm}$ ) in which the tag was measured and decoded with background calibration. The filled squares correspond to the positions where the tag was successfully read. We can see there is a region around $x=0$ and $y<0$ in which there are no correct readings of the tag, this corresponds to where the mechanic arm was over the antenna during the 'empty' measurement, which is highlighted in Fig. 4(a). Once this object is no longer at the same position, this decoding approach deteriorates.

For example, in Fig. 5(a) the TD signal of backgroundcalibrated measurement performed at position $(-2,-2,10)$ can be seen (in blue), in this case, it is clear that the calibration was not effective to remove the large first pulse due to the initial reflection, and the result is an incorrect reading of the tag in that position, as seen in Fig. 5(b) (blue curve), hence the need for time-gating to increase the system's performance, as further discussed in the next section.

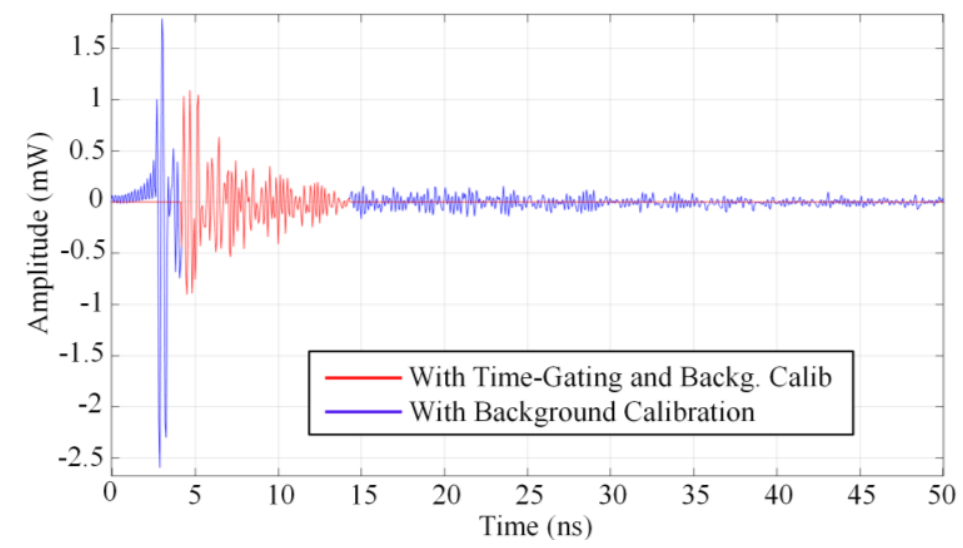

(a)

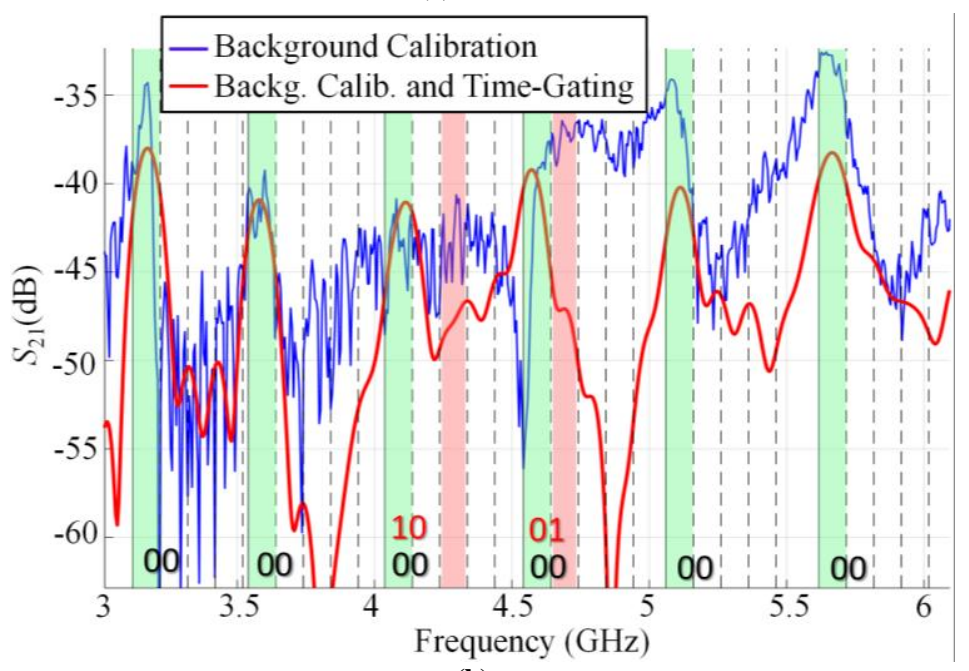

(b)

Fig. 5. Example of time signal with time-gating (in red) and without (in blue). Measurement taken at position $(-2,-2,10) \mathrm{cm}$. (a) Time signal, window starting at $t_{\text {start }}=2.5 \mathrm{~ns}$ and ending at $t_{\text {stop }}=12.5 \mathrm{~ns}$. (b) Frequency representation, with correct code detected (black numbers) with time-gating and incorrect code detected with only background calibration (red numbers). 
Background calibration is then applied on all 29,791 measurements within the $30 \times 30 \times 30 \mathrm{~cm}^{3}$ volume. Fig. 6 (a) presents the $3 \mathrm{D}$ reading performance of the decoding with background calibration. The color scheme indicates the success rate obtained in each z-plane, blue being the lowest values obtained and red the highest. The obtained success rate in full volume is $7.29 \%$. The maximum success rate of $13.84 \%$ is obtained at $z=12 \mathrm{~cm}$.
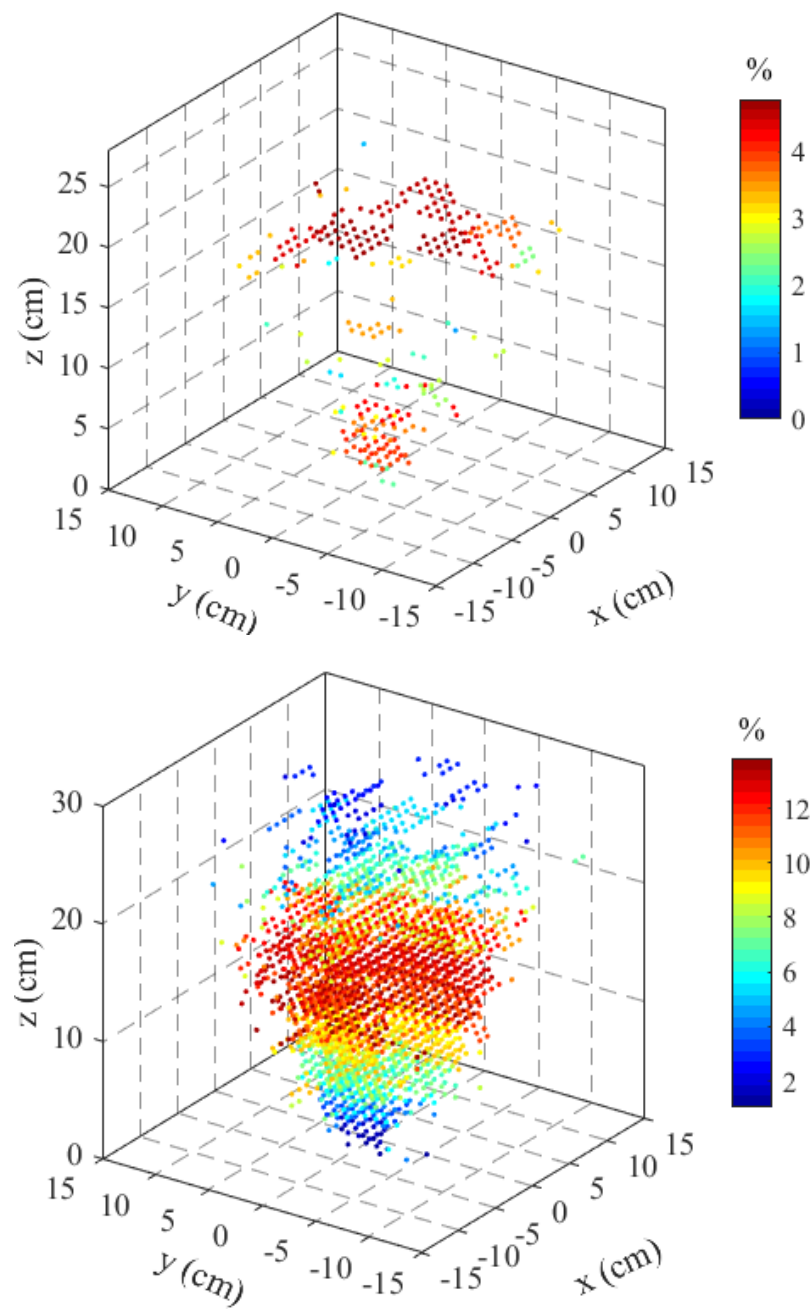

(b)

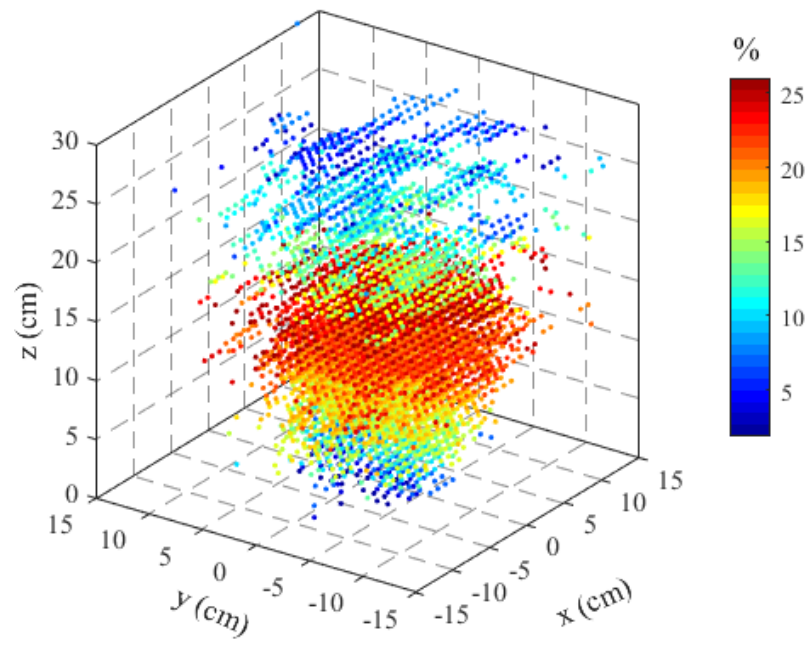

(a)

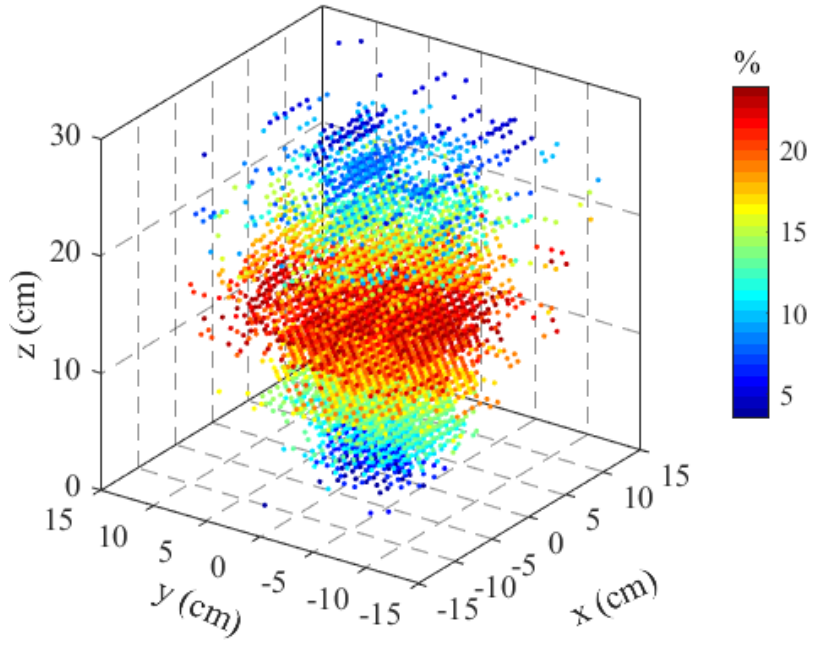

(d)

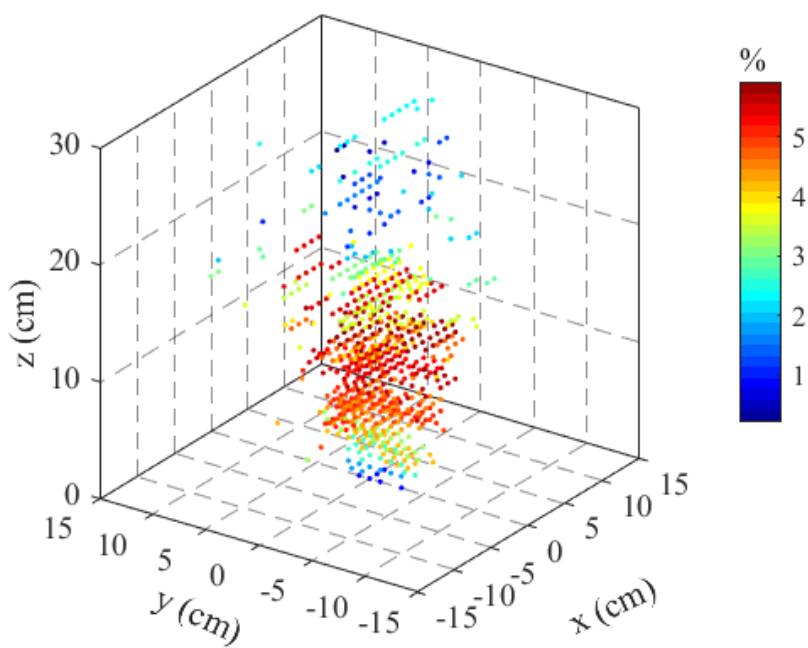

(e)

Fig. 6. 3D Success Rate inside $30 \times 30 \times 30 \mathrm{~cm}^{3}$ volume using different decoding methods. The color scheme indicates the success rate for each $x y$ plane: (a) Raw Amplitude; (b) Background Calibration; (c) Background Calibration and Time-Gating; (d) Background Calibration and STFT; (e) Background Calibration and STPA.

\section{B. Time-Gating}

The second method consists of employing time-gating onto the background-calibrated signals. Since we used a VNA, the $S_{21}$ was measured, and thus it is given as a set of amplitude values for each frequency. To perform time-gating we must extract the signal's IFFT, to obtain its's TD representation, and then select a time-window from this signal, to which the Fast Fourier Transform (FFT) is applied in order to bring it back to the FD.

(c) The backscattered signal from a chipless tag is a combined response including the antenna coupling, the tag's substrate, the tag's scatterer and the background (see [41]). With a static background, the background subtraction procedure can merely mitigate the antenna coupling and the response from the static objects. In practical cases, the backscattered signal part from the mobile objects is very difficult to be removed by background subtraction. Also, the background subtraction procedure might not help in removing the direct optical reflections [initial pulse caused by the tag's substrate as shown 
in Fig. 5(a)]. This structure mode of the chipless RFID tag causes the dislocation of the peak apexes, which ultimately causes the unsuccessful decoding [see blue line and red areas in Fig. 5(b)]. It is important to note that we have used only one clutter signal measured at $(0,0,15)$ for the background calibration of all the tag measurements of 29,791 positions. For this reason, some of part of clutter is still present even after the background calibration. It is why, the application of the time-windowing on the background-calibrated signals produces the successful decoding [see red line and green areas in Fig. 5(b)].

The window used for time-gating was a simple rectangular window, with sharp transition, corresponding to a rectangle function starting at time $t_{\text {start }}$ and stopping at $t_{\text {start }}+T$, as defined by

$$
\operatorname{rect}\left(\frac{t-t_{\text {start }}}{T}-\frac{1}{2}\right)=\left\{\begin{array}{c}
1, t_{\text {start }} \leq t \leq t_{\text {start }}+T \\
0, \text { elsewhere }
\end{array}\right.
$$

It has been optimized to increase the overall decoding success considering all the measurement results. We point out that a 'success' is determined by the correct reading of the 12 bits, and the term success rate is used here to indicate the percentage of positions in which the tag is read. Thus, we have calculated the success rate inside the total volume for various different time-windows applied, and selected the best window, which is applied for all the measurements.

Fig. 7 shows different success rates as a function of the beginning time, $t_{\text {start }}$, for different window durations, $T=6$ ns, $8 \mathrm{~ns}, 10 \mathrm{~ns}$ and $12 \mathrm{~ns}$. Note that the optimal $t_{\text {start }}$ obtained was $2.75 \mathrm{~ns}$ for a window duration $T=10 \mathrm{~ns}$.

For the environment we have presented, the larger the window, higher is the success rate. Nonetheless, for durations larger than $10 \mathrm{~ns}$, the increment is no longer significant. However, for harsher environments - for example with the presence of more objects and closer walls - multipath reflections can occur, so shorter window duration would have to be considered.

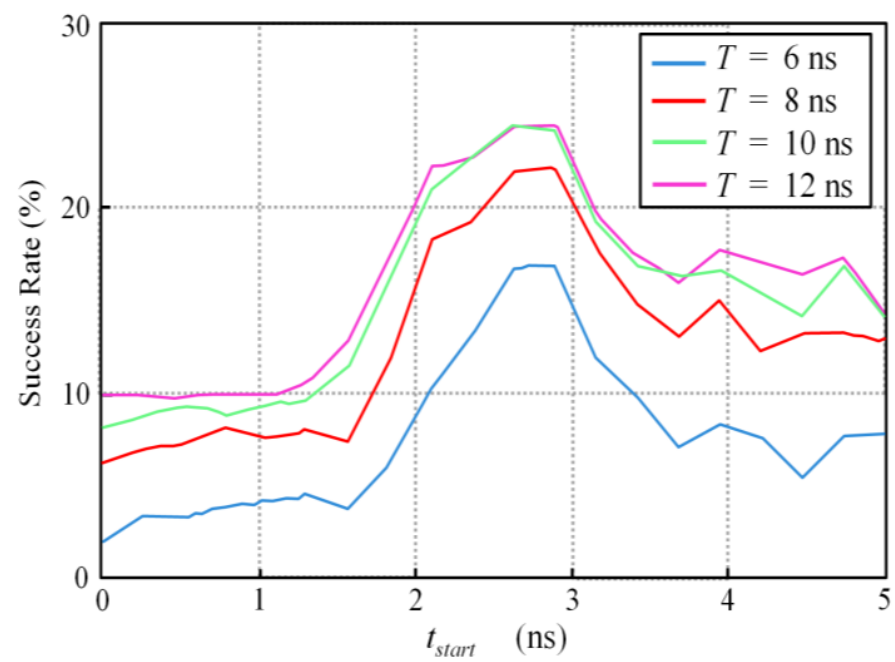

Fig. 7. Optimization study of the window used for time-gating, in terms of success rate and $t_{\text {start }}$, for different window durations $(T=6 \mathrm{~ns}, 8 \mathrm{~ns}, 10 \mathrm{~ns}, 12$ ns). Optimal $t_{\text {start }}$ obtained is $2.75 \mathrm{~ns}$ and $T=10 \mathrm{~ns}$.
An example of a TD signal measured at position $(-2,-2,10) \mathrm{cm}$ can be seen in Fig. 5(a). The blue curve corresponds to the full TD signal - only with background calibration - whereas the red curve corresponds to the timegating signal. It can be seen that the chosen window excludes the first part of the signal, which corresponds to the structure mode, and thus contains no information. This part is not excluded just by performing the background calibration, since it is due to the reflection on the tag.

In Fig. 5(b) the response in frequency for the same case is shown, the blue curve corresponds to the decoding without time-gating whereas the red curve is the decoding with timegating. We can see that the signal with time-gating is significantly smoother, with less noise, and that it is correctly decoded at the given position, whereas the decoding with only background calibration results in an error, with the $2^{\text {nd }}$ and $3^{\text {rd }}$ resonant peaks being mistakenly detected in different subbands, indicated in red. The erroneous decoding yields ID = 00.00 .10 .01 .00 .00 .

Fig. 4 shows the read success rate for the plane $z=10 \mathrm{~cm}$, using only background calibration [Fig. 4(a)] and background calibration and time-gating [Fig. 4(b)]. We can see that the 'blind spot' around $x=0$ and $y<0$, in Fig. 4(a) is corrected with the use of the optimized time-gating. Comparing Fig. 4(a) and 4(b), it can be seen that with time-gating, the area where the tag is correctly identified is larger, which means a greater success rate. For this particular reading plane, the rate increases from $9.68 \%$ to $24.1 \%$ with addition of time-gating. We also notice that the area with time-gating is more symmetrical in relation to the antenna's center, approximating a circle.

Time-gating is then applied on all 29,791 measurements, with background calibration, within the $30 \times 30 \times 30 \mathrm{~cm}^{3}$ volume. Fig. 6(c) presents the 3D reading performance of the decoding with time-gating. The obtained success rate in full volume is $14.99 \%$. The maximum success rate of $26.01 \%$ is obtained at $z=16 \mathrm{~cm}$.

\section{2-DIMENSIONAL DECODING APPROACHES}

In this Section, we present the implementation of two 2-D time- and frequency-based decoding approaches: STFT and STPA.

\section{A. Short Time Fourier Transform}

To implement the temporal separation based STFT averaging decoding method [58] for each axial displacement, the VNA-measured FD signal is transformed into a TD signal using IFFT. This TD signal is terminated at $100 \mathrm{~ns}$. Then, STFT is calculated with a sliding hamming window $w(\tau)$ using FFT. Parameter $\tau$ is the time index of the sliding hamming window, $w(\tau)$. Finally, averaging along the time axis is performed. For the background-calibrated signal measured at position $(0,0,10) \mathrm{cm}$, Fig. 8 shows the calculated STFT with a sliding hamming window $w(\tau)$ of $14 \mathrm{~ns}$. The reason behind this choice of $14 \mathrm{~ns}$ for the length of $w(\tau)$ will be explained later in this Section. 


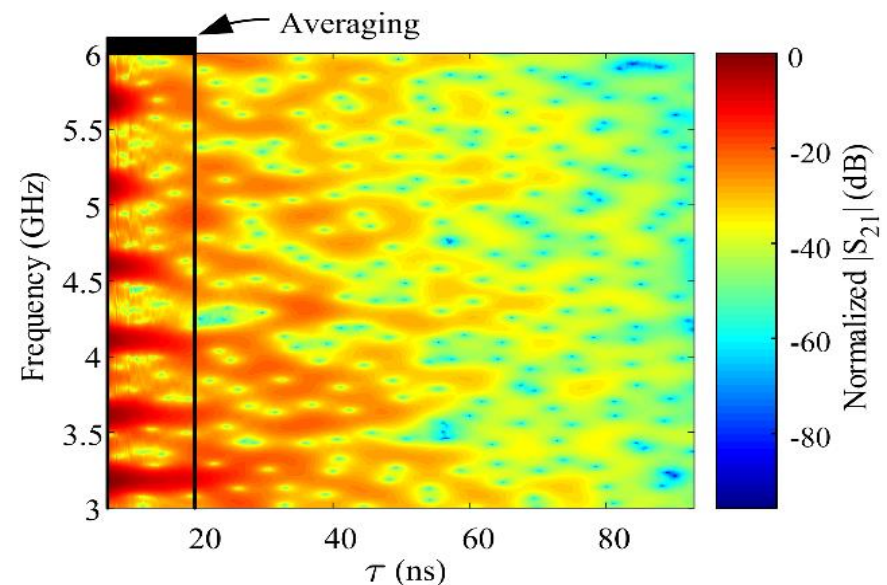

Fig. 8. Calculated STFT for calibrated signal $\left(S_{21}^{\text {backg.calib }}\right)$ measured at $(0,0$, 10) $\mathrm{cm}$ with a sliding hamming window $w(\tau)$ of $14 \mathrm{~ns}$.

In Fig. 8, the black marked region is the averaging window. The start time $t_{\text {start }}^{\text {avg }}$ of the averaging window can be chosen at early time instants of $\tau$. The stop time $t_{\text {stop }}^{\text {avg }}$ of the averaging window is estimated based on the operating parameters of the scatterer exhibiting maximum quality factor, $Q_{\max }$, in the design of chipless RFID tag: the frequency of resonance $f_{\text {rmax }}$ and $Q_{\max }$, equal to $3.59 \mathrm{GHz}$ and 97.1 , respectively, which correspond to the second resonator. The selection of stop time $t_{\text {stop }}^{\text {avg }}$ is more significant than $t_{\text {start }}^{\text {avg }}$, as an optimum choice of

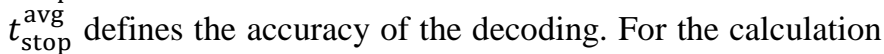
of $t_{\text {stop }}^{\text {avg }}$, first a decaying exponential envelope based on $f_{\text {rmax }}$ and $Q_{\max }, A_{e}=e^{-\left(\pi f_{\operatorname{rmax}} / Q_{\max }\right) \tau}$, is generated. Then $t_{\text {stop }}^{\text {avg }}$ is selected such that $t_{\text {stop }}^{\text {avg }}=\left.\tau\right|_{A_{e}=A_{e}^{\text {thresh }}}$, where $A_{e}^{\text {thresh }}$ is a threshold amplitude level. This procedure is shown in Fig. 9, in which the calculation of two values $t_{\mathrm{stop} 1}^{\mathrm{avg}}$ and $t_{\mathrm{stop2}}^{\mathrm{avg}}$ with $A_{e}^{\text {thresh }}=10^{-1}$ and $A_{e}^{\text {thresh }}=10^{-3}$ is presented, respectively.

For the STFT presented in Fig. 8, two averaged signals are calculated using two values $t_{\text {stop1 }}^{\text {avg }}$ and $t_{\text {stop2 }}^{\text {avg and shown in }}$ Fig. 10. It can be observed that the averaged signal calculated with $t_{\text {stop1 }}^{\text {avg }}$ (solid line) presents better selectivity than the averaged signal calculated with $t_{\text {stop2 }}^{\text {avg }}$ (dashed line). This better selectivity is due to the higher SNR at $t_{\text {stop } 1 \text {. The }}^{\text {avg }}$ significance of $t_{\text {stop1 }}^{\text {avg }}$ will also be highlighted later in this Section. For the rest of this Section, unless the contrary is stated, the STFT averaging method is applied using $t_{\text {stop } 1}^{\text {avg }}$.

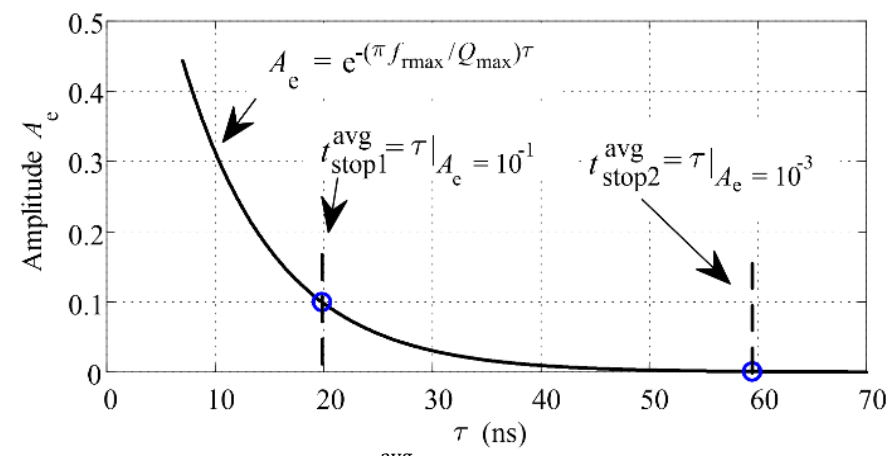

Fig. 9. Calculation of stop time $t_{\text {stop }}^{\text {avg }}$ of averaging window.

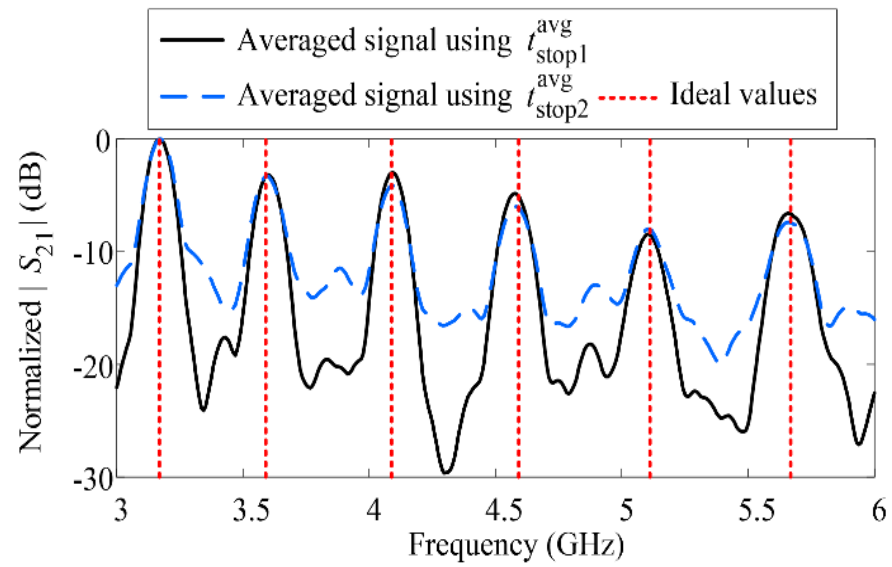

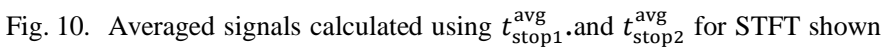
in Fig. 8.

The resolution of time and frequency is dependent on the proper choice of the length of $w(\tau)$ [58]. Fig. 11 presents the effect of the length of $w(\tau)$ on the reading performance of STFT averaging method in the given volume. For both background-calibrated signals $\left(S_{21}^{\text {backg.calib }}\right)$ and raw uncalibrated signals $\left(S_{21}^{\text {tag }}\right)$, the reading performance improves from $11.92 \%$ to $14.93 \%$ and from $5.73 \%$ to $8.89 \%$, respectively, as the length of $w(\tau)$ increases from 9 ns to 14 ns. Subsequently, the reading performance falls to $11.5 \%$ and $6.22 \%$ at the length of $w(\tau)$ equal to $18 \mathrm{~ns}$ for backgroundcalibrated signals and raw uncalibrated signals, respectively. It is found that a value of $14 \mathrm{~ns}$ is an optimum choice for the length of $w(\tau)$. For this reason, the length of $w(\tau)=14 \mathrm{~ns}$ is used in the implementation of STFT averaging decoding method.

The STFT averaging decoding method is applied on all 29,791 background-calibrated measurements, within the $30 \times 30 \times 30 \mathrm{~cm}^{3}$ volume. Fig. $6(\mathrm{~d})$ presents the $3 \mathrm{D}$ reading performance of this decoding. The obtained success rate in full volume is $14.93 \%$. The maximum success rate of $23.93 \%$ is obtained at $z=15 \mathrm{~cm}$.

If we were to use $t_{\text {stop2 }}^{\text {avg }}$ for the calculation of the averaging window, the success rate in full volume would be $8.18 \%$, showing that the selected $t_{\mathrm{stop} 1}^{\mathrm{avg}}$ is an optimum choice for the calculation of averaging window.

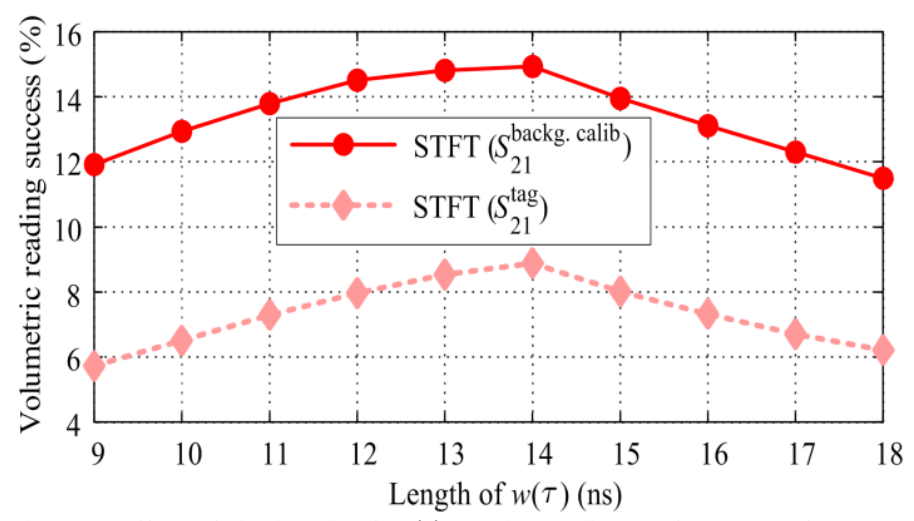

Fig. 11. Effect of the length of $w(\tau)$ on the reading performance of STFT averaging method in the $30 \times 30 \times 30 \mathrm{~cm}^{3}$ volume. 


\section{B. Short Time Prony Analysis}

Singularity expansion method (SEM) was proposed by C. E. Baum in 1971 [59]. This method has widely been used for radar target detection [60]. In TD, Prony analysis is one of the techniques to implement SEM. Prony analysis was introduced by Prony in [61] and later this method was improved in [62] to make it less sensitive to the noise. In the literature, Prony analysis has been employed to analyze and detect power systems' signals [63], radar targets [64], buried ordnance [65], plane wave transmissions into dispersive half-space [66] and noisy antenna signals [67].

In this paper, for the first time, we have implemented STPA as a decoding method for chipless RFID technology. In this regard, for each axial displacement, the VNA-measured FD signal is transformed into TD signal using IFFT. This TD signal is terminated at $20 \mathrm{~ns}$. Then, STPA is calculated using sliding hamming window $w(\tau)$ using Prony analysis algorithm (see [67] and [68]). In Prony analysis algorithm, we have taken the number of complex poles $M=30$. For estimated complex poles at each $\tau$ of $w(\tau)$, a pole-filtering procedure is carried out: 1) neglecting the complex poles that do not present their complex conjugates; 2) neglecting the complex conjugate poles; 3) neglecting the non-decaying poles. For the background-calibrated signal measured at $(0,0$, 10) $\mathrm{cm}$, Fig. 12(a) shows the calculated STPA with a sliding hamming window $w(\tau)$ of $14 \mathrm{~ns}$. The reason behind this choice of $14 \mathrm{~ns}$ is that the achieved reading performance is maximal at this value (as with the STFT averaging method). The effect of the length of $w(\tau)$ on the reading performance of STPA will be shown later in this Section.

From all calculated poles, a histogram is generated, and the mean values of maximum probable bins are selected as the decoded signal, as shown in Fig. 12(b). The width of each histogram bin is $10 \mathrm{MHz}$.

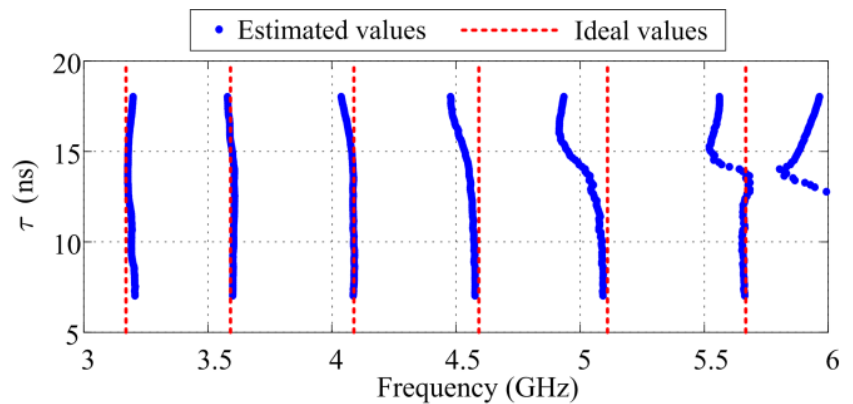

(a)

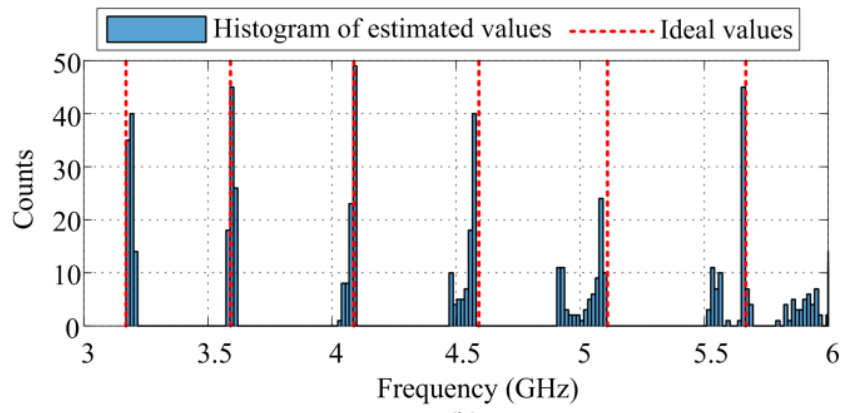

(b)

Fig. 12. Implementation of STPA with a sliding hamming window $w(\tau)$ of 14 ns: (a) Estimated poles; (b) Decoded ID.
STPA is applied to all 29,791 background-calibrated signals within the given volume. Fig. 6(e) presents the 3D reading performance of this method. The obtained success rate in full volume is $2.4 \%$. The maximum success rate of $5.93 \%$ is obtained at $z=9 \mathrm{~cm}$. For STPA applied to raw uncalibrated signals, the obtained success rate in full volume is lower, $2.01 \%$.

Fig. 13 presents the effect of the length of $w(\tau)$ on the reading performance of STPA inside the given volume. For the background-calibrated signals $\left(S_{21}^{\text {backg.calib }}\right)$ the reading performance improves from $1.1 \%$ to $2.4 \%$, whereas for the raw signals $\left(S_{21}^{\mathrm{tag}}\right)$, it improves from $0.62 \%$ to $2.01 \%$, when the length of $w(\tau)$ increases from 9 ns to $14 \mathrm{~ns}$. Subsequently, the reading performance falls to $\approx 0$ at the length of $w(\tau)=18 \mathrm{~ns}$ for both background-calibrated and uncalibrated signals. As for STFT decoding, the value of $14 \mathrm{~ns}$ is found an optimum choice for the length of $w(\tau)$. For the length of $w(\tau)$ larger than $14 \mathrm{~ns}$, the TD signal is terminated at $40 \mathrm{~ns}$. This is done to make the length of the TD signal sufficiently larger than the length of $w(\tau)$.

\section{RESUlTS AND DisCUSSION}

In this section we present the results for the decoding of the tag inside the $30 \times 30 \times 30 \mathrm{~cm}^{3}$ volume, comparing the different decoding methods for all 29,791 measurements performed.

Fig. 6(a-e) show the comparison between 3D reading performances for each of the selected decoding methods. The presence of the markers corresponds to the correct readings, whereas the absence of a marker indicates positions where it was erroneously decoded. The color scheme serves to indicate the success rate obtained in each $z$-plane, blue being the lowest values obtained and red the highest.

Adjoined to this, Table I summarizes a comparison of success rates for several distances with each method. For all cases, the reading volumes and success rates were obtained with the optimal parameters presented, when applicable. That is, for time-gating, the window used had parameters $t_{\text {start }}=$ $2.75 \mathrm{~ns}$ and duration $T=10 \mathrm{~ns}$; and for both STFT and STPA, the window had width $w(\tau)=14 \mathrm{~ns}$.

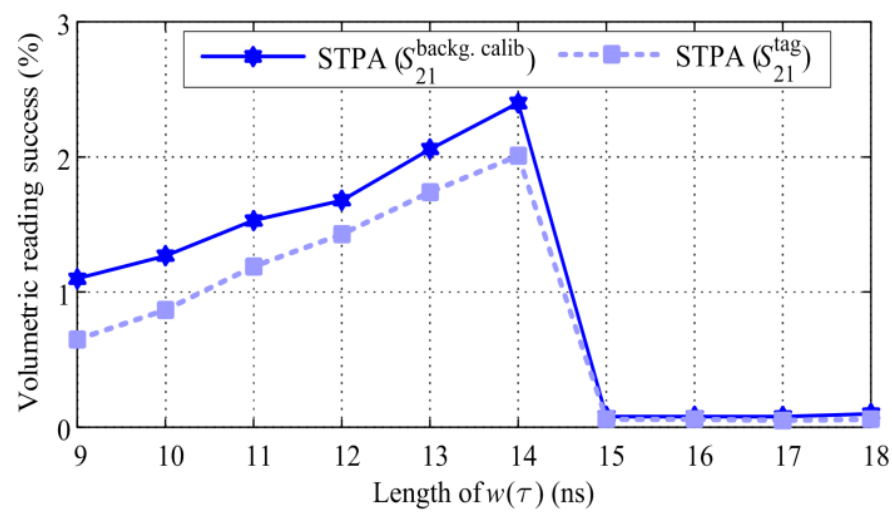

Fig. 13. Effect of the length of $w(\tau)$ on the reading performance of STPA in the $30 \times 30 \times 30 \mathrm{~cm}^{3}$ volume. 


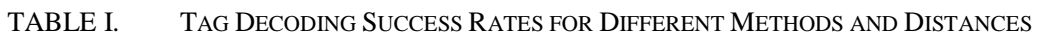

\begin{tabular}{|c|c|c|c|c|c|c|c|c|c|}
\hline & \multirow{2}{*}{$\begin{array}{r}\text { Success } \\
\text { Rate }(\%)\end{array}$} & \multicolumn{6}{|c|}{$\mathbf{z}(\mathbf{c m})$} & \multirow[b]{2}{*}{$\begin{array}{l}\text { Success in } \\
\text { Total Volume } \\
(\%)\end{array}$} & \multirow[b]{2}{*}{$\begin{array}{c}\text { Distance with } \\
\text { best success rat }\end{array}$} \\
\hline & & 5 & 10 & 15 & 20 & 25 & 30 & & \\
\hline \multirow{3}{*}{ 1-D } & $\begin{array}{l}\text { Raw - Amplitude } \\
\text { Decoding }\end{array}$ & 1.77 & 0.21 & 0.21 & 0.52 & 0 & 0 & 0.87 & $4.79 \%$ at $19 \mathrm{~cm}$ \\
\hline & Background Calibration & 7.18 & 9.68 & 13.63 & 10.51 & 3.85 & 2.39 & 7.29 & $13.84 \%$ at $12 \mathrm{~cm}$ \\
\hline & $\begin{array}{l}\text { Backg. Calibr. with } \\
\text { Time-gating }\end{array}$ & 17.69 & 24.14 & 25.49 & 14.98 & 9.16 & 7.80 & 14.99 & $26.01 \%$ at $16 \mathrm{~cm}$ \\
\hline \multirow{4}{*}{ 2-D } & Raw - STFT & 12.49 & 14.05 & 15.50 & 6.97 & 2.39 & 0.73 & 8.89 & $16.65 \%$ at $12 \mathrm{~cm}$ \\
\hline & Raw-STPA & 3.54 & 5.72 & 1.98 & 0.21 & 0 & 0 & 2.01 & $6.35 \%$ at $9 \mathrm{~cm}$ \\
\hline & $\begin{array}{l}\text { Backg. Calibr. with } \\
\text { STFT }\end{array}$ & 15.40 & 20.19 & 23.93 & 18.42 & 8.12 & 4.79 & 14.93 & $23.93 \%$ at $15 \mathrm{~cm}$ \\
\hline & $\begin{array}{l}\text { Backg. Calibr. with } \\
\text { STPA }\end{array}$ & 3.64 & 4.68 & 2.81 & 0.83 & 0.73 & 1.04 & 2.40 & $5.93 \%$ at $9 \mathrm{~cm}$ \\
\hline
\end{tabular}

Firstly, it can be seen that the reading successes are more frequent around the central axis, in front of the antenna. We point out that, for all the methods shown, when the tag is very close to the antenna (e.g., $z=1 \mathrm{~cm})$, the area in which it is successfully decoded is small and centred directly in front of the antenna. As the tag gets farther away, the corresponding reading area increases. However, after $z \geq 15 \mathrm{~cm}$, although the area continues to expand, the decoding successes are less dense, for both methods. This is because the reading volume is linked to the radiation pattern of the antenna (as shown in [69]), with a narrow aperture angle and with reflected power decreasing (lower SNR) as the tag is positioned farther from the antenna. Another way to express this is that the reading performance improves as the reading distance increases from the near-field region to the Fresnel region and declines as the reading distance is approaching the far-field region.

By comparing background calibration to time-gating and STFT decoding of calibrated measurements [Fig. 6(b), 6(c) and 6(d), respectively], we can see that time-gating and STFT improve drastically the performance of the system, yielding a larger reading volume, which is denser and reaches farther outwards, in the $x$ - and $y$ - directions. Overall, the reading volume is more than doubled when we compare time-gating and STFT to background calibration (over 2 times the number of successes inside the same volume).

As for the STPA method, the reading successes are strictly confined near the center of the given volume. The reading performance of STPA is less than the reading performance of the other methods, advantageous only in relation to the raw amplitude decoding.

The best read distance varied according to the method applied, going from $9 \mathrm{~cm}$ to $19 \mathrm{~cm}$ (last column of Table I). Time-gating and STFT had best read distance around 15-16 $\mathrm{cm}$.

We have seen that the optimized time-gating of the calibrated measurements is the best decoding method from those analyzed, being slightly better than STFT (see Table I), with the advantage that it is a 1-D approach, less complex than STFT.
For close ranges (up to $10 \mathrm{~cm}$ ), time-gating is 2.5 times better than background calibration, and for longer ranges (around $30 \mathrm{~cm}$ ), it is over 3 times better than the simple background calibration, and over 1.5 times better than STFT. STFT only showed better performance compared to timegating at $z=20 \mathrm{~cm}$.

We should also point out that the use of time-gating and STFT notably improves the decoding for positions around $x=$ 0 and $y<0$, which correspond to situations when the mechanic arm would be over the antenna, causing undesirable reflections, which strongly deteriorate the decoding.

The use of the appropriate decoding method is essential to allow the chipless RFID system to be used in real applications and environments, guaranteeing a better performance in terms of reading range and reading volume.

\section{CONCLUSIONS}

In this paper, we have shown a comparison between 1-D and 2-D decoding methods for a Chipless RFID system comprised of a 12-bit FC depolarizing tag being measured inside a $30 \times 30 \times 30 \mathrm{~cm}^{3}$ volume in a real environment.

For the first time, STPA has been implemented as a decoding method for chipless RFID, and its performance discussed.

The system's performance was optimized based on the practical measurements performed, to achieve the highest success rate inside the given volume. That is, we have obtained the optimal parameters for the window used for timegating, and for the averaging windows used for STFT and STPA methods.

We have found the time-gating method and STFT provide the same performance for reading the chipless RFID tag since they can remove the structural part of the tag response and double the success rate, when compared to background calibration. Also, time-gating benefits from a low computational complexity which makes it a good option for real implementation. 


\section{REFERENCES}

[1] Jie Gao, Zhibo Pang, Qiang Chen, and Li-Rong Zheng, "Interactive packaging solutions based on RFID technology and Controlled Delamination Material," in IEEE Int. Conf. on RFID (IEEE RFID), 2010, pp. 158-165.

[2] L. R. Carley, G. Colak, L. Chomas, L. Pileggi, and K. Mai, "Technologies for secure RFID authentication of medicinal pills and capsules," in IEEE Int. Conf. on RFID Technol. and Appl. (RFID-TA), 2016, pp. 10-15.

[3] Horng-Lin Shieh, Shih-Fong Lin, and Wen-Sheng Chang, "RFID medicine management system," in Int. Conf. on Mach. Learn. and Cybern., 2012, vol. 5, pp. 1890-1894.

[4] M. Kärkkäinen and T. Ala-Risku, "Automatic identification applications and technologies," in Logistics Res. Netw. Annu. Conf., 2003.

[5] M. Al-Zewairi, J. Alqatawna, and O. Al-Kadi, "Privacy and security for RFID Access Control Systems: RFID Access Control Systems without back-end database," in IEEE Jordan Conf. on Appl. Elect. Eng. and Comput. Technol. (AEECT), 2011, pp. 1-6.

[6] W. M. Adbulkawi and A.-F. A. Sheta, "Printable Chipless RFID Tags for IoT Applications," in 1st Int. Conf. on Comput. Appl. Inf. Secur. (ICCAIS), 2018, pp. 1-4.

[7] K. Yang, U. Botero, H. Shen, D. Forte, and M. Tehranipoor, "A split manufacturing approach for unclonable chipless RFIDs for pharmaceutical supply chain security," in Asian Hardware Oriented Secur. and Trust Symp. (AsianHOST), 2017, pp. 61-66.

[8] R. Want, "An introduction to RFID technology," IEEE Pervasive Comput., vol. 5, no. 1, pp. 25-33, Jan. 2006.

[9] S. Genovesi, F. Costa, A. Monorchio, and G. Manara, "Phase-only encoding for novel chipless RFID tag," in IEEE RFID Technol. and Appl. Conf. (RFID-TA), 2014, pp. 68-71.

[10] R. Rezaiesarlak and M. Manteghi, Chipless RFID: Design Procedure and Detection Technique. New York, USA: Springer, 2015.

[11] T. Athauda and N. Karmakar, "Screen Printed Chipless RFID Resonator Design for Remote Sensing Applications," in Asia-Pacific Microw. Conf. (APMC), 2018, pp. 1321-1323.

[12] S. Merilampi, V. Ihanakangas, and J. Virkki, "User-driven development with scientific applied $\mathrm{x}$ - RFID-controlled physiogame case study," in IEEE Int. Conf. on RFID Technol. and Appl. (RFIDTA), 2019, pp. 167-170.

[13] H. Takahata, F. Kojima, M. Okada, T. Sugiura, T. Sato, and O. Oshiro, "Thoracoscopic surgery support system using passive RFID marker," in Annu. Int. Conf. of the IEEE Eng. in Medicine and Biol. Soc., 2012, pp. 183-186.

[14] V. Palazzi, F. Gelati, U. Vaglioni, F. Alimenti, P. Mezzanotte, and L. Roselli, "Leaf-Compatible Autonomous RFID-Based Wireless Temperature Sensors for Precision Agriculture," in IEEE Topical Conf. on Wireless Sensors and Sensor Netw. (WiSNet), 2019, pp. 1-4.

[15] "RFID FAQs - Barcoding, Inc. - Barcoding, Inc." [Online]. Available: https://www.barcoding.com/resources/frequently-asked-questionsfaq/rfid-faqs/. [Accessed: 27-Feb-2020].

[16] A. Vena, E. Perret, and S. Tedjini, "Chipless RFID Tag Using Hybrid Coding Technique," IEEE Trans. Microw. Theory Tech., vol. 59, no. 12, pp. 3356-3364, Dec. 2011.

[17] E. Perret, Radio Frequency Identification and Sensors: From RFID to Chipless RFID. New Jersey: John Wiley \& Sons, 2014.

[18] M. Garbati, E. Perret, and R. Siragusa, Chipless RFID Reader Design for Ultra-Wideband Technology: Design, Realization and Characterization. London, UK: ISTE Press - Elsevier, 2018.

[19] A. Vena, E. Perret, and S. Tedjni, "A Depolarizing Chipless RFID Tag for Robust Detection and Its FCC Compliant UWB Reading System," IEEE Trans. Microw. Theory Tech., vol. 61, no. 8, pp. 2982-2994, Aug. 2013.

[20] S. Fan, T. Chang, X. Liu, Y. Fan, and M. M. Tentzeris, "A Depolarizing Chipless RFID Tag with Humidity Sensing Capability," in IEEE Int. Symp. on Antennas and Propag. USNC/URSI Nat. Radio Sci. Meeting, 2018, pp. 2469-2470.

[21] F. Babaeian and N. Karmakar, "A Cross-Polar Orientation Insensitive Chipless RFID Tag," in IEEE Int. Conf. on RFID Technol. and Appl. (RFID-TA), 2019, pp. 116-119.

[22] M. Khaliel, A. Fawky, A. El-Awamry, A.-E. Mahmoud, and T. Kaiser, "Printable, high coding capacity chipless RFID tags for lowcost item tagging," in IEEE 14th Int. Conf. on Netw. Sens. and Control (ICNSC), 2017, pp. 351-355
[23] A. Habib, H. Anam, Y. Amin, and H. Tenhunen, "High-density compact chipless RFID tag for item-level tagging," in Int. Appl. Comput. Electromagn. Soc. Symp. (ACES), 2018, pp. 1-2.

[24] T. Cheng et al., "Design of UWB Chipless RFID Tag using Microstrip and Slot Cross-shaped Resonators," in Int. Flexible Electron. Technol. Conf. (IFETC), 2018, pp. 1-4.

[25] Md. A. Islam and N. Karmakar, "On a compact printable dualpolarized chipless RFID tag using slot length variation encoding technique for barcode replacement," in IEEE MTT-S Int. Microw. Symp., 2015, pp. 1-4.

[26] Md. A. Islam, Y. Yap, and N. Karmakar, “' $\Delta$ ' slotted compact printable orientation insensitive chipless RFID tag for long range applications," in 9th Int. Conf. on Elect. and Comput. Eng. (ICECE), 2016, pp. 283-286.

[27] A. Vena, E. Perret, and S. Tedjini, "High-Capacity Chipless RFID Tag Insensitive to the Polarization," IEEE Trans. Antennas Propag., vol. 60, no. 10, pp. 4509-4515, Oct. 2012.

[28] A. Vena, L. Sydänheimo, L. Ukkonen, and M. M. Tentzeris, "A fully inkjet-printed chipless RFID gas and temperature sensor on paper," in IEEE RFID Technol. and Appl. Conf. (RFID-TA), 2014, pp. 115-120.

[29] R. Nair et al., "A fully printed passive chipless RFID tag for low-cost mass production," in 8th Eur. Conf. on Antennas and Propag. (EuCAP 2014), 2014, pp. 2950-2954.

[30] Z. Li and S. Bhadra, "A Flexible Printed Complementary Split-Ring Resonator Based Chipless RFID," in 18th Int. Symp. on Antenna Technol. and Appl. Electromagn. (ANTEM), 2018, pp. 1-2.

[31] G. S. Vardhan, N. Sivadasan, and A. Dutta, "QR-code based chipless RFID system for unique identification," in IEEE Int. Conf. on RFID Technol. and Appl. (RFID-TA), 2016, pp. 35-39.

[32] S. Genovesi, F. Costa, M. Borgese, F. A. Dicandia, A. Monorchio, and G. Manara, "Chipless RFID sensor for rotation monitoring," in IEEE Int. Conf. on RFID Technol. Application (RFID-TA), 2017, pp. 233236.

[33] A. Guillet, A. Vena, E. Perret, and S. Tedjini, "Design of a chipless RFID sensor for water level detection," in 15th Int. Symp. on Antenna Technol. and Appl. Electromagn., 2012, pp. 1-4.

[34] R. Suwalak, C. Phongcharoenpanich, P. Akkaraekthalin, and D. Torrungrueng, "Curved Meander Line Resonators for Chipless RFID Sensors," in IEEE Int. Conf. on RFID Technol. and Appl. (RFID-TA), 2019, pp. 26-28.

[35] T. Athauda and N. C. Karmakar, "The Realization of Chipless RFID Resonator for Multiple Physical Parameter Sensing," IEEE Internet Things J., vol. 6, no. 3, pp. 5387-5396, Jun. 2019.

[36] A. M. J. Marindra and G. Y. Tian, "Multiresonance Chipless RFID Sensor Tag for Metal Defect Characterization Using Principal Component Analysis," IEEE Sens. J., vol. 19, no. 18, pp. 8037-8046, Sep. 2019.

[37] S. Tedjini, O. Boularess, T. Andriamiharivolamena, H. Rmili, and T. Aguili, "A novel design of chipless RFID tags based on alphabets," in IEEE MTT-S Int. Microw. Symp. (IMS), 2017, pp. 1561-1563.

[38] R. T. de Alencar, N. Barbot, M. Garbati, and E. Perret, "Practical Comparison of Decoding Methods for Chipless RFID System in Real Environment," in IEEE Int. Conf. on RFID Technol. and Appl. (RFIDTA), 2019, pp. 207-211.

[39] S. Preradovic, I. Balbin, N. C. Karmakar, and G. Swiegers, "A Novel Chipless RFID System Based on Planar Multiresonators for Barcode Replacement," in IEEE Int. Conf. on RFID, 2008, pp. 289-296.

[40] A. Vena, E. Perret, and S. Tedjini, Chipless RFID based on RF Encoding Particle: Realization, Coding and Reading System. Elsevier, 2016.

[41] A. Ramos, A. Lazaro, R. Villarino, and D. Girbau, "Time-domain UWB RFID tags for smart floor applications," in IEEE RFID Technol. and Appl. Conf. (RFID-TA), 2014, pp. 165-169.

[42] C. S. Hartmann, "A global SAW ID tag with large data capacity," in IEEE Ultrason. Symp., 2002. Proceedings., 2002, vol. 1, pp. 65-69 vol.1.

[43] C. Herrojo et al., "Time-Domain-Signature Chipless RFID Tags: Near-Field Chipless-RFID Systems With High Data Capacity," IEEE Microw. Mag., vol. 20, no. 12, pp. 87-101, Dec. 2019.

[44] M. G. Pettus, "RFID system utilizing parametric reflective technology," US7460014B2, 02-Dec-2008.

[45] W. A. Linton, J. Linton, J. E. Krueger, and M. P. Dearing, "Radio frequency identification method and system of distributing products," US7258276B2, 21-Aug-2007. 
[46] D. H. Nguyen, M. Zomorrodi, and N. C. Karmakar, "Spatial-Based Chipless RFID System," IEEE J. Radio Freq. Identif., vol. 3, no. 1, pp. 46-55, Mar. 2019.

[47] M. Zomorrodi and Nemai. C. Karmakar, "Image-based chipless RFID system with high content capacity for low cost tagging," in IEEE Int. Microw. and RF Conf. (IMaRC), 2014, pp. 41-44.

[48] M. Pöpperl, J. Adametz, and M. Vossiek, "Polarimetric Radar Barcode: A Novel Chipless RFID Concept With High Data Capacity and Ultimate Tag Robustness," IEEE Trans. Microw. Theory Tech., vol. 64, no. 11, pp. 3686-3694, Nov. 2016.

[49] F. Bonnefoy et al., "Identification of random internal structuring $\mathrm{THz}$ tags using images correlation and SIWPD analysis," in 44th Int. Conf. on Infrared, Millimeter, and Terahertz Waves (IRMMW-THz), 2019, pp. $1-1$.

[50] C. Herrojo, F. Paredes, and F. Martín, "A new paradigm in chiplessRFID: all-dielectric permittivity contrast tags," in IEEE Int. Conf. on RFID Technol. and Appl. (RFID-TA), 2019, pp. 163-166.

[51] F. Babaeian and N. C. Karmakar, "Hybrid Chipless RFID Tags- A Pathway to EPC Global Standard," IEEE Access, vol. 6, pp. 6741567426, 2018.

[52] C. Feng, W. Zhang, L. Li, L. Han, X. Chen, and R. Ma, "Angle-Based Chipless RFID Tag With High Capacity and Insensitivity to Polarization," IEEE Trans. Antennas Propag., vol. 63, no. 4, pp. 1789-1797, Apr. 2015.

[53] O. Rance, R. Siragusa, P. Lemaître-Auger, and E. Perret, "Toward RCS Magnitude Level Coding for Chipless RFID," IEEE Trans. Microw. Theory Tech., vol. 64, no. 7, pp. 2315-2325, Jul. 2016.

[54] O. Rance, R. Siragusa, P. Lemaître-Auger, and E. Perret, "RCS magnitude coding for chipless RFID based on depolarizing tag," in IEEE MTT-S Int. Microw. Symp., 2015, pp. 1-4.

[55] G. C. Wan, Q. Gu, X. R. Zhang, and M. S. Tong, "Frequency-coded chipless RFID tag based on hybrid coding technique," in IEEE Int. Symp. on Antennas and Propag. USNC/URSI Nat. Radio Sci. Meeting, 2017, pp. 2519-2520.

[56] A. Jiménez-Sáez, M. Schüßler, M. Nickel, and R. Jakoby, "Hybrid time-frequency modulation scheme for chipless wireless identification and sensing," in IEEE SENSORS, 2017, pp. 1-3.

[57] R. Nair, E. Perret, and S. Tedjini, "Chipless RFID based on group delay encoding," in IEEE Int. Conf. on RFID Technol. and Appl., 2011, pp. 214-218.

[58] A. Ramos, E. Perret, O. Rance, S. Tedjini, A. Lázaro, and D. Girbau, "Temporal Separation Detection for Chipless Depolarizing Frequency-Coded RFID," IEEE Trans. Microw. Theory Tech., vol. 64, no. 7, pp. 2326-2337, Jul. 2016.

[59] C. E. Baum, "On the singularity expansion method for the solution of electromagnetic interaction problems," Air Force Weapons Laboratory, Kirkland AFB, New Mexico, Note 8, 1971.

[60] C. E. Baum, E. J. Rothwell, K.-M. Chen, and D. P. Nyquist, "The singularity expansion method and its application to target identification," Proc. IEEE, vol. 79, no. 10, pp. 1481-1492, Oct. 1991.

[61] R. Prony, "Essai expérimental et analytique sur les lois de la dilatabilité de fluides élastiques et sur celles de la force expansive de la vapeur d'alcool," Journal de l'Ecole Polytechnique, Paris, pp. $1440-1454,1795$.

[62] M. D. Rahman and Kai-Bor Yu, "Total least squares approach for frequency estimation using linear prediction," IEEE Trans. Acoust. Speech Signal Process., vol. 35, no. 10, pp. 1440-1454, Oct. 1987.

[63] J. F. Hauer, C. J. Demeure, and L. L. Scharf, "Initial results in Prony analysis of power system response signals," IEEE Trans. Power Syst., vol. 5, no. 1, pp. 80-89, Feb. 1990.

[64] R. Carriere and R. L. Moses, "High resolution radar target modeling using a modified Prony estimator," IEEE Trans. Antennas Propag., vol. 40, no. 1, pp. 13-18, Jan. 1992.

[65] Chi-Chih Chen and L. Peters, "Buried unexploded ordnance identification via complex natural resonances," IEEE Trans. Antennas Propag., vol. 45, no. 11, pp. 1645-1654, Nov. 1997.

[66] A. M. Attiya, "Transmission of Pulsed Plane Wave Into Dispersive Half-Space: Prony's Method Approximation," IEEE Trans. Antennas Propag., vol. 59, no. 1, pp. 324-327, Jan. 2011.

[67] F. Sarrazin, A. Sharaiha, P. Pouliguen, J. Chauveau, S. Collardey, and P. Potier, "Comparison between Matrix Pencil and Prony methods applied on noisy antenna responses," in Loughborough Antennas Propag. Conf., 2011, pp. 1-4.
[68] L. L. Grant and M. L. Crow, "Comparison of Matrix Pencil and Prony methods for power system modal analysis of noisy signals," in North Amer. Power Symp., 2011, pp. 1-7.

[69] R. T. de Alencar, N. Barbot, M. Garbati, and E. Perret, "Characterization of Chipless RFID Tag in a 3-Dimensional Reading Zone," in IEEE Int. Symp. on Antennas and Propag. and USNC-URSI Radio Sci. Meeting, 2019, pp. 639-640. 